\title{
Structural health monitoring system using piezoelectric networks with tuned lamb waves
}

\author{
Bruno Rocha ${ }^{\mathrm{a}}$, Carlos Silva ${ }^{\mathrm{b}}$ and Afzal Suleman ${ }^{\mathrm{a}, *}$ \\ a IDMEC/IST - Instituto Superior Tecnico, Av. Rovisco Pais, Lisbon, Portugal \\ ${ }^{\mathrm{b}}$ Aeronautical Laboratory, Portuguese Air Force Academy, BA1, Granja do Marques, Pero Pinheiro, Sintra, \\ Portugal
}

\begin{abstract}
The paper presents a structural health monitoring system based on propagation of tuned Lamb waves and their interference with discontinuities. The dispersion curves are studied to determine the appropriate type and dimension of transducers and to select the optimum scanning frequencies and relevant propagation modes. A piezoelectric sensor network was implemented in an aluminum plate in order to generate and to sense the wave propagation and associated reflections. The algorithm developed for damage detection relies on the comparison of undamaged and damaged responses of the structure. Combinations of filters and statistical methods were applied to detect differences in the sensor signals acquired for the two different states (damaged and undamaged), corresponding to damage reflections. In order to eliminate the false positives due to noise, a probability analysis is performed to obtain the final damage position. The software designed for the current application allows the automatic calculation of dispersion curves, it executes the scans, performs data processing, executes the detection algorithm and presents the probable damages and their positions in a graphical form. Experiments were performed with the introduction of cumulative damages in the plate such as surface and through-the-thickness holes and cuts, ranging from $7 \mathrm{~mm}$ to $1 \mathrm{~mm}$ in diameter. Additionally, a stringer was attached to the plate by a single rivet line to simulate an aircraft skin structure. Cuts originating from rivet holes and connecting adjacent rivets, as well as loosened rivets were detected by the system. The introduction of the stringer resulted in a loss of precision in the determination of the radial position of the damages near it. Also, the network revealed significant difficulties in the detection of damages beyond the stringer.
\end{abstract}

Keywords: Structural health monitoring, non-destructive testing and evaluation, lamb waves, piezoelectric networks

\section{Introduction}

In the early years of aviation, structures were designed to have an infinite life, by assuring that their operation stress limits were within their fatigue stress limits. This approach resulted in heavier structures that strongly conditioned the aircraft's available payload. Further studies on the fatigue phenomena led to the application of a Safe Life approach. A finite service life was established within which the probability of fatigue crack initiation and growth was improbable. The Safe Life concept was used for many years, however it failed in the sense that manufacture defects were unaccounted for. Other types of damage, which could strongly reduce component life, such as corrosion or accidental damage were also not accounted for. Frequently, service loads did not comply with the design loads, damage models were inaccurate and stress analysis was inadequately applied. Such errors resulted in multiple accidents such as the Havilland COMETs between May 1952 and January 1954. The last one flew only 1000 flights, while the simulation tests predicted 3060 flights during its operational life.

Progress achieved in crack growth studies led to the development of the Fail Safe concept. In this approach, a structure retained a residual strength, without repair, after failure or partial failure of a primary structural element. Structural design was based on multiple load paths with the simultaneous implementation of crack stoppers. Under

\footnotetext{
${ }^{*}$ Corresponding author. Professor, University of Victoria, Victoria, Canada. E-mail: suleman@uvic.ca.
} 


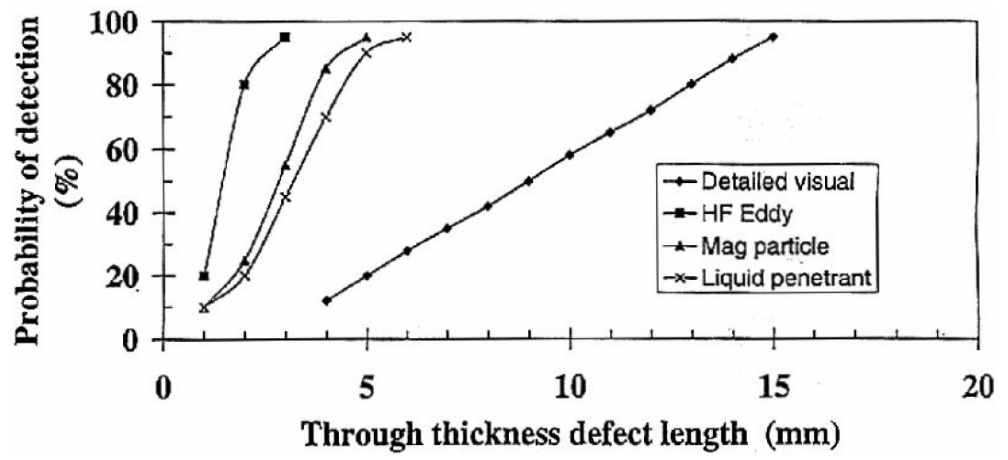

Fig. 1. Probability of Detection at 95\% confidence of selected NDT [1].

these conditions, despite crack initiation, stress levels were kept low, contributing to a slow crack propagation, so that it could supposedly be detected prior to failure. This concept was the origin for the Damage Tolerance philosophy, presently regulated by the Federal Aviation Regulations (FAR25) and Joint Aviation Regulations (JAR25.571). Here, quantitative crack growth calculations are made assuming worst case scenarios, including initial manufacturing defects and sensitivity details about the crack detection capabilities of the different inspection methods. The service life until first inspection is defined and, in order to avoid catastrophic failure, an inspection and maintenance schedule is defined. Presently, only engine pylons and landing gears are designed according to the Safe Life concept.

Fundamental for the practicality of the Damage Tolerance philosophy is the application of different types of NonDestructive Test and Evaluation (NDT\&E). NDT consists of inspecting components without inflicting permanent damage to them. NDE is a term that is often used interchangeably with NDT. However, technically, NDE is used to describe structural assessment that is more quantitative in nature. For example, a NDE method would not only locate a defect, but it would also be used to measure something about that defect such as its size, shape, or orientation. There are several inspection methods, namely: Visual Testing (VT); Penetrant Liquid Testing (LPT); Magnetic Particle Testing (MT); Electromagnetic Testing (ET) or Eddy Current Testing; Radiography (RT); Ultrasonic Testing (UT), etc. The component type, its assembled or disassembled status, its material, geometry, the type of damages to be searched, etc, are some of the aspects that define which inspection method is better suited and should be applied. The Probability of Detection (PoD) of different defect dimensions, for some of the methods previously referred, is presented in Fig. 1. Except for visual inspections, most of the methods achieve a sufficient PoD for defects larger than $5 \mathrm{~mm}$ ( $2 \mathrm{~mm}$ considering Eddy Current testing). A competitive inspection technique should be able to detect defects of 1 to $2 \mathrm{~mm}$ with a PoD of $80 \%$ or higher.

Presently, all the above referenced inspection methods are not embedded into the structures, cannot be applied in real time and are localized inspection methods. Aircraft structures must undergo a strict inspection and maintenance scheduled plan. A Predictive maintenance approach does not assure total operation safety. Unpredicted damages (such as impact damages) might occur and develop to catastrophic dimensions without being detected, between scheduled maintenance operations. Smaller damages in areas of difficult access might not be detected or correctly assessed during inspections. Predictive maintenance also leads to high operation costs since parts can be replaced just because their predicted lifetime was exceeded, even if inspected without revealing any damage. Moreover, related disassembling and assembling operations are usually time-consuming, requiring the aircraft to be grounded. A grounded aircraft is not profitable and in fact it generates additional costs, related to hangar time, maintenance time and operations, leasing, etc. The objectives of the proposed SHM technology is to detect damages, and subsequently assess the position, shape and size of the damage. Furthermore, during the operation life of the structure, the growth rate of damages can be measured and the remaining life can be estimated in real-time.

\section{Lamb waves - background}

In 1917, Horace Lamb [2] predicted analytically the existence of a particular type of acoustic waves in solids called Lamb waves. His study was based in the theory of Rayleigh waves, which are mechanical elastic waves in solids, 
near a free boundary [3]. Lamb waves exist in thin plate like structural components, in the presence of two parallel free boundaries, and their propagation direction is parallel to the component's mid surface. Due to the low damping imposed by the free boundaries to the propagation of these waves, one of their most interesting characteristic is their ability to propagate to long distances with minor amplitude damping. In 1950, Mindlin [4] completed a theoretical approach to this type of waves. At that time, progress related with Lamb waves was motivated by their medical applications in World War II.

In 1961, Worlton [5] suggested the potential of applying Lamb waves for structural inspection and damage detection, and a new NDE potential technique emerged. The interesting behavior of Lamb waves is their interference with damages (and boundaries and other material or geometry discontinuities), generating reflections that can be detected.

The prospective damage types prone to be detected by this kind of inspection were summarized by Rose [6]. Advantages of this method included: inspection of large structures while retaining their coating and insulation, e.g. a pipe system under water; exceptional sensitivity to multiple defects with high identification accuracy; small energy consumption and considerable cost-effectiveness.

Worden et al. [7] established the axioms for every SHM system. Naturally, the first one refers that every damage assessment is based on a comparative analysis, between damage and undamaged states. The second states that in unsupervised mode, only damage existence and location can be determined. To identify damage type and severity, supervised modes must be implemented. The remaining three are related to operation conditions, environmental conditions, sensitivity, noise, frequency and damage size.

Presently, SHM methodologies are focused mainly on high frequency waves and vibration analysis, since one of the principles is that the damage size that the method is able to detect is inversely proportional to the frequency applied. Damage detection by high frequency waves is achieved through, between others, the emission of Lamb waves and gathering of the structural responses to it. Basically it is a pulse-echo scanning method. It can be mainly applied to beams and plates, what is extremely useful for Aerospace structures, since most of them can be decomposed in these basic structural components. In the presence of a damage, reflections will occur. Through the detection of reflections as differences between the damage and undamaged responses, it is possible to determine the existence of damage. Determining the difference in time between the emission and the echo, and knowing the propagation speed, the position of the damage can then be determined.

Lamb waves are omnidirectional when emitted by just one actuator, but they can be steered into one preferential direction using an actuator array. Implementing a specified delay between actuators, individual waves will (positively) interfere in certain positions, creating a wave front propagating into a certain direction.

Important and inter related aspects that must be considered in the application of a tuned Lamb wave based inspection method are the type of sensors/actuators to be employed and how they are fitted into the structure; the actuation type; the propagation characteristics in the host medium; and finally, the type of signal processing and evaluation to be applied [8].

Research is being conducted in different fronts. The behavior of Lamb waves generation and propagation and the transducer influence on such behavior, applying numerical methods and experimentation, was investigated [9]. Lamb wave emission experiments in aluminum plates proved that these high frequency waves were sensitive to damage presence, especially when small distances between damage and probe were considered [10]. Several of these experiments proved that more than one mode could be efficiently excited and applied in damage detection. By being frequency selective in the generation of the waves, a particular mode could be stimulated. It was also observed that different types of damages were better detected by different wave modes - interior damages to the structure were better detected when symmetric or compressive waves were stimulated, while surface damages were better detected when anti-symmetric or flexure waves were emitted. Depending on the number of inflection points across the material thickness, different $\mathrm{S}$ or $\mathrm{A}$ wave modes might appear. These are designated by $\mathrm{S}_{n}$ and $\mathrm{A}_{n}$ waves, being $\mathrm{n}$ the number of inflection points across thickness. In Fig. 2 the primary modes, $\mathrm{S}_{0}$ and $\mathrm{A}_{0}$, are presented.

While the $\mathrm{S}$ mode essentially produces compression and traction movements, the A mode produces movement in the normal direction with respect to the propagation direction (the mid surface). The upper, mid and lower surfaces move in phase.

One other important characteristic of Lamb waves yet to refer is their dispersive behavior - i.e. the dependence of their propagation speed to their frequency - and the simultaneous excitation of multiple modes in certain frequencies. 

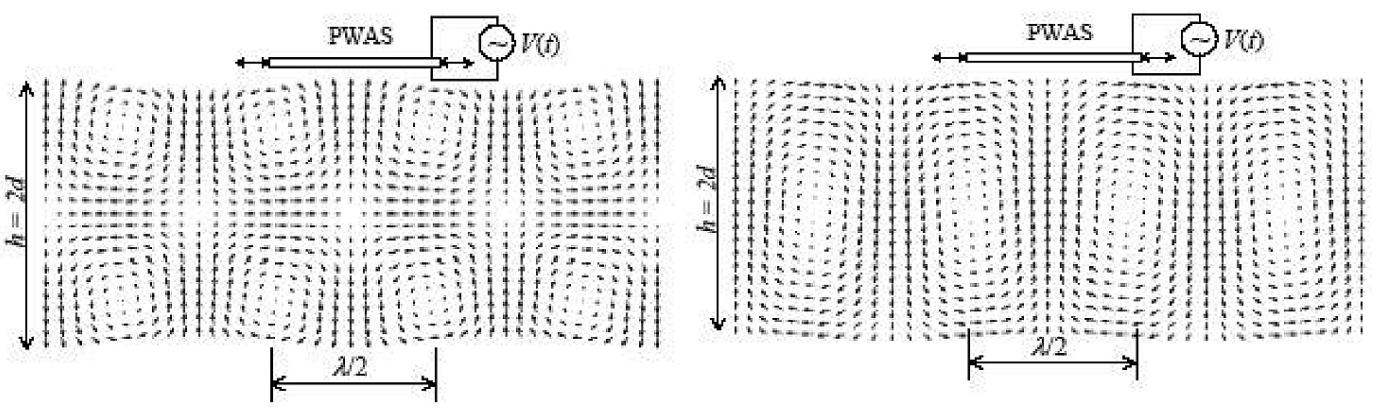

Fig. 2. Symmetric wave $\left(\mathrm{S}_{0}\right)$ and Anti-Symmetric wave $\left(\mathrm{A}_{0}\right)[11]$.

Since this can introduce major difficulties in the application of Lamb waves for structural inspection, it is also a topic under intense research. As an example, experimental results were attained using 2D Fast Fourier Transform (FFT) on data collected using a vibrometer [12].

Numerical models for wave propagation, damage modeling and the interference in between them are being developed, using Finite Element Analysis (FEA). Between different Finite Element Methods applied, interesting results were attained with the ones based on Spectral Finite Elements [13].

As an emerging technique, the concern on reliability, in the application of systems based in Lamb waves, and their associated aspects and components, spurred other investigations. Durability of actuators [14] and their response under extreme conditions such as high temperature, large strain and cryogenic environments [15] were the theme for different studies.

Piezoelectric transducers have been selected in most cases to generate the waves. They have the capability to work under high frequencies, performing both actuator and sensor tasks. This is the main reason for their systematic application for in situ health monitoring [16]. Their size, shape and location must be carefully selected in order to achieve the desired results. Giurgiutiu was able to carry out experiments using tuned guided waves with PZT arrays [17].

The potential of Lamb wave based SHM methodologies to monitor large metallic aircraft surfaces was depicted by Dalton et al. [18]. Practical considerations concerning the painting, sealant application and thickness changes in structural panels were presented. More recently, J. Michaels and T. Michaels [19] developed an imaging signal processing technique to detect damage position. An aircraft fuselage panel was tested successfully, using two strips of PZTs, by Ihn and Chang [20]. A beam forming approach was implemented on a wing panel with satisfying results [21]. Ultimately, all these different approaches will have to be subjected to real flight tests. As an example, experiments applying different inspection methodologies were performed during flight tests, under harsh flight conditions, using a UK Hawk MK-1A, with promising results [22].

\section{Lamb waves' dispersion curves}

The Lamb wave phase velocity, $\mathrm{C}_{L}$, depends on frequency - dispersive behavior referred before, wave mode considered, host material mechanical properties and thickness, h. One straightforward conclusion is that, if multiple excitation frequencies are applied in the wave generation, packs of waves with different propagation velocities will be generated, introducing noise and added difficulty in the damage detection method. Moreover, for a given actuation frequency, multiple modes may be excited and the generated wave will be a complex combination of different modes, raising problems in the evaluation of sensors' signals. The dispersion curves depict the various existing modes and their propagation velocities for each frequency of excitation. Therefore, it was of the outmost importance to start by calculating and plotting the dispersion curves. An optimum range of excitation frequencies could be determined to minimize the number of different wave modes to be excited and then attain a good separation in terms of propagation speeds for the different excited modes. To obtain the dispersion curves it is necessary to calculate the Lame constants, $\mathrm{C}_{S}$ and $\mathrm{C}_{P}$. 


$$
\begin{aligned}
C_{S} & =\sqrt{\frac{E}{2 \rho(1+v)}} \\
C_{P} & =\sqrt{\frac{v E}{\rho(1+v)(1-2 v)}+\frac{E}{\rho(1+v)}}
\end{aligned}
$$

Defining $\xi, \zeta$ and $\bar{d}$ as

$$
\begin{gathered}
\xi=\frac{C_{S}}{C_{P}} \\
\zeta=\frac{C_{S}}{C_{L}} \\
\bar{d}=\frac{w}{C_{S}} d,
\end{gathered}
$$

where $w$ is the frequency in $\mathrm{rad} / \mathrm{s}$ and $d$ is the plate's half thickness.

The dispersion curves can then be calculated by solving the Rayleigh-Lamb frequency equations. For the symmetrical mode the equation becomes

$$
\frac{\tan \left(\sqrt{1-\zeta^{2}} \bar{d}\right)}{\tan \left(\sqrt{\xi^{2}-\zeta^{2} \bar{d}}\right)}=4 \zeta^{2} \sqrt{1-\zeta^{2}} \frac{\sqrt{\xi^{2}-\zeta^{2}}}{\left(2 \zeta^{2}-1\right)^{2}}
$$

While for the anti-symmetrical mode it becomes

$$
\frac{\tan \left(\sqrt{1-\zeta^{2}} \bar{d}\right)}{\tan \left(\sqrt{\xi^{2}-\zeta^{2} \bar{d}}\right)}=\frac{\left(2 \zeta^{2}-1\right)^{2}}{4 \zeta^{2} \sqrt{1-\zeta^{2}} \sqrt{\xi^{2}-\zeta^{2}}}
$$

Both equations have several roots, corresponding to different symmetric and anti-symmetric Lamb wave modes: $\mathbf{S}_{0}$, $\mathrm{A}_{0}, \mathrm{~S}_{1}, \mathrm{~A}_{1}$, etc. Solving numerically these equations in order to $\mathrm{C}_{L}$ and $f$, knowing that

$$
f=\frac{w}{2 \pi}
$$

The evolution of the roots, i.e. the dispersion curves, is obtained - Fig. 3. In the calculation, the plate thickness and host material were specified - an aluminum plate with the characteristics presented in Table 1 was considered.

At low frequencies $(f<500 \mathrm{kHz})$ the propagation speed of the symmetric Lamb wave approaches the speed of axial waves, $C_{P}$, which for the material and plate thickness selected approaches $5500 \mathrm{~m} / \mathrm{s}$. Also, the derivative of this propagation speed with frequency is relatively small. The propagation speed of the anti-symmetric waves tends to zero as the excitation frequency is decreased.

Lamb waves travel in packs. In order to determine the pack velocity, a group velocity, $\mathrm{C}_{g}$, has to be defined,

$$
C_{g}=C_{L}^{2}\left[C_{L}-(f d) \frac{d C_{L}}{d(f d)}\right]^{-1}
$$

To simplify the $\mathrm{C}_{g}$ calculation, the following approximation can be established

$$
\frac{d C_{L}}{d f} \approx \frac{\Delta C_{L}}{\Delta f}
$$

The curves plotted in Fig. 4 were calculated using this approximation and applying a numerical method to compute the solution for $\mathrm{C}_{g} \mathrm{Vs} f$. Actually these are the velocities that can be measured and applied for SHM purposes.

Although the dispersion curves were computed up to scanning frequencies of 3.5MHZ, only the curves corresponding to frequencies in the range up to $1 \mathrm{MHz}$ are shown. For emission frequencies higher than $1 \mathrm{MHZ}$, other higher (inflection) wave modes than $\mathrm{S}_{0}$ and $\mathrm{A}_{0}$, and with different propagation velocities, exist. While the higher modes 
Table 1

$1 \mathrm{~m}$ square aluminum plate properties

\begin{tabular}{lc}
\hline Property & Value \\
\hline Young modulus $(\mathrm{E})$ & $70 \mathrm{GPa}$ \\
Density $(\rho)$ & $2700 \mathrm{Kg} / \mathrm{m}^{3}$ \\
Poison ratio $(v)$ & 0.35 \\
Thickness $(\mathrm{h})$ & $0.002 \mathrm{~m}$ \\
\hline
\end{tabular}

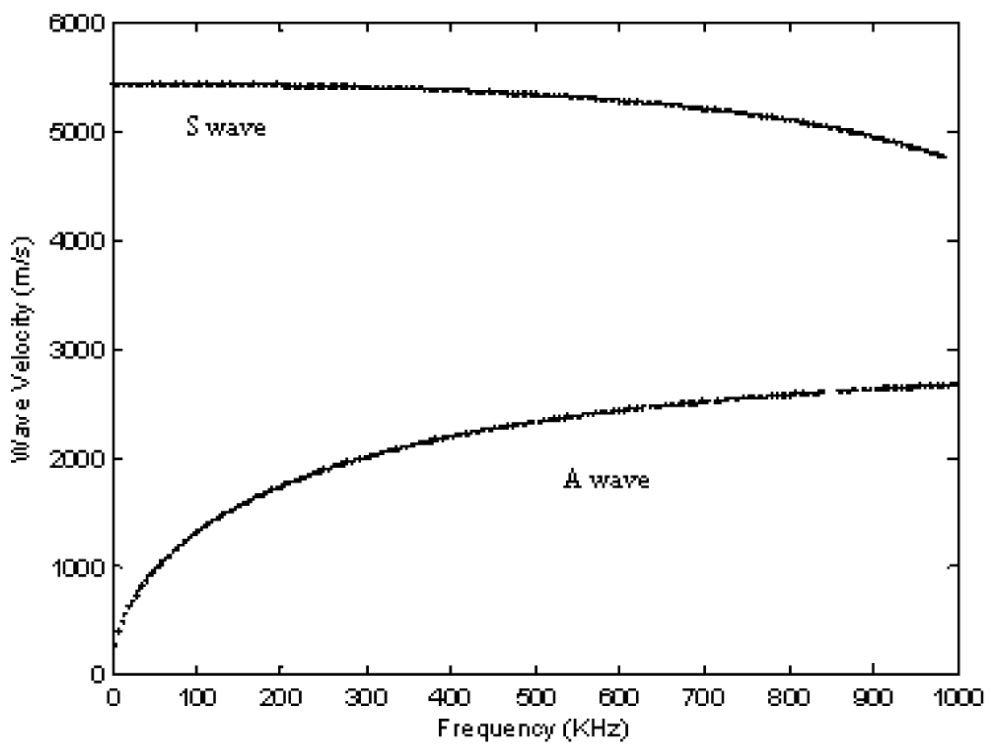

Fig. 3. Phase velocities for a $2 \mathrm{~mm}$ thick aluminum plate.

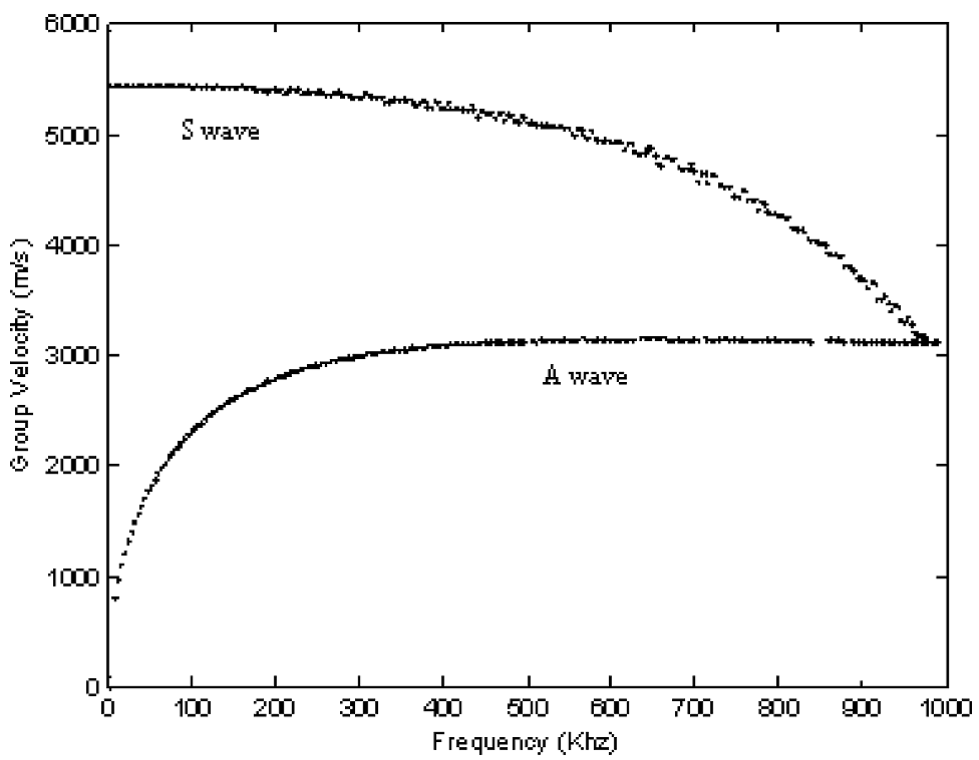

Fig. 4. Group velocities for a $2 \mathrm{~mm}$ thick aluminum plate. 


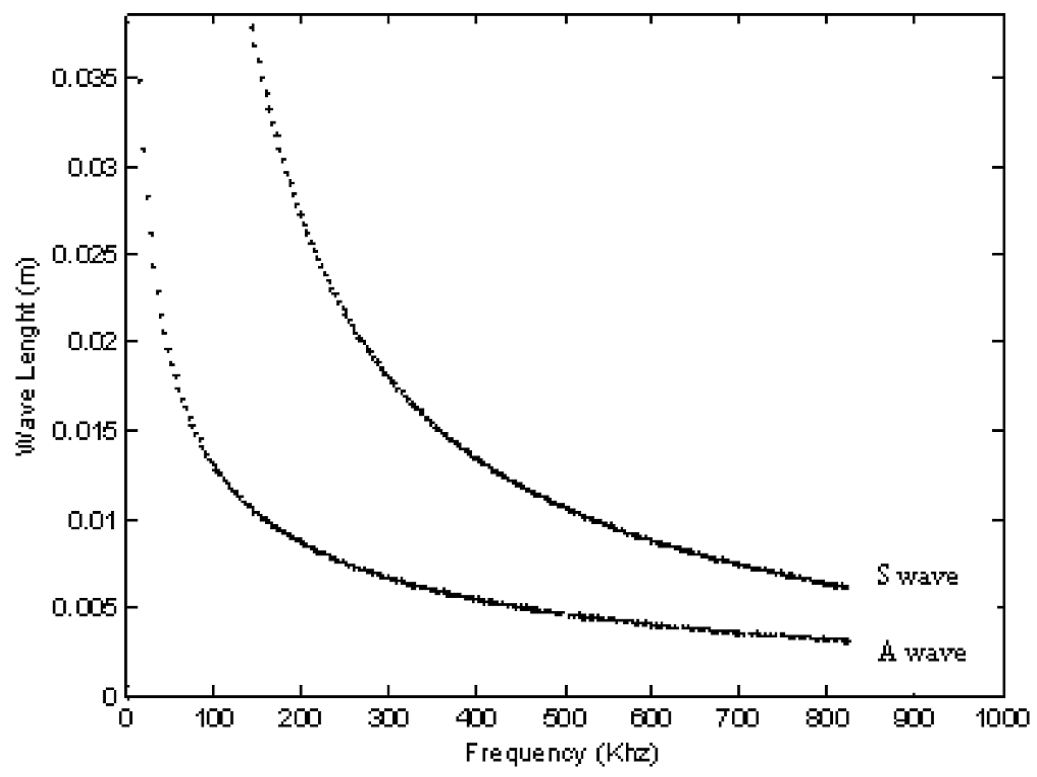

Fig. 5. Lamb waves wavelength vs frequency.

are not fundamental for the implementation of damage detection algorithms, a demarked complexity is introduced in terms of the propagated combined wave morphology and consequently acquired sensor signal. Such introduces considerable difficulties in the decomposition of the signal to detect and assess the intended possible differences corresponding to reflections. In Fig. 3 it may also be observed the approximation of the propagation phase velocities of the $\mathrm{S}_{0}$ and $\mathrm{A}_{0}$ wave modes as scanning frequency increases. At high frequencies $(f>2500 \mathrm{kHz})$, the dispersion curves for the $S_{0}$ and $A_{0}$ modes coalesce, while the group velocities for the two main modes intersect for frequencies around $1 \mathrm{MHz}-$ Fig. 4. It can be noted also an increasing difference in between phase and group velocities for the symmetric primary mode with increasing scanning frequency, approaching $1 \mathrm{MHz}$. The differences between phase and group velocities result in the stretching of the wave pack (with corresponding stretching in time of the correspondent sensor signal), while closer propagation speeds of different wave modes result in smaller differences in time between their corresponding sensor signals. This might lead to an increased complexity in the sensors signals due to poor time separation between the different modes (to the point of superposition of the corresponding sensed signals for the different wave modes, when similar propagation speeds are reached). These observations result in the selection of an interesting scanning frequency range up to $700 \mathrm{kHz}$, where sufficient separation between the propagation speeds of the two different modes is observed.

Based on the phase velocity dispersion curves, i.e. the scanning frequency and the corresponding wave propagation velocity, it is possible to calculate the relation between the wavelength of the emitted wave and its frequency. These curves are presented in Fig. 5.

Considering symmetric, or traction-compression waves, to correctly actuate and sense wave induced strains, transducers should have a dimension inferior to half the wavelength, as stated by Giurgiutiu [17]. As can be deduced from Fig. 2, when the transducer's diameter is half of the wavelength, it is expected that transducers will be subjected to the highest compression and traction strain rates, presenting the highest amplitude in their signal (simultaneously maximizing the dimension of the sensor and consequently their potential output).

The scanning wavelength to frequency relation limits the dimension of transducers to be applied. Further limitations must be considered due to the relation between the dimensions and the natural frequencies of PZT transducers. At the same time the installation of transducers 'footprint' should be reduced as much as possible - in between others to diminish their influence in Lamb wave propagation, decreasing their influence in the local stiffness and thickness increase.

However, when anti-symmetric, or flexural waves are considered, as can be observed in Fig. 2, transducers will be subjected to higher strains when their diameter is similar to the wavelength. The preferential generation of symmetric 

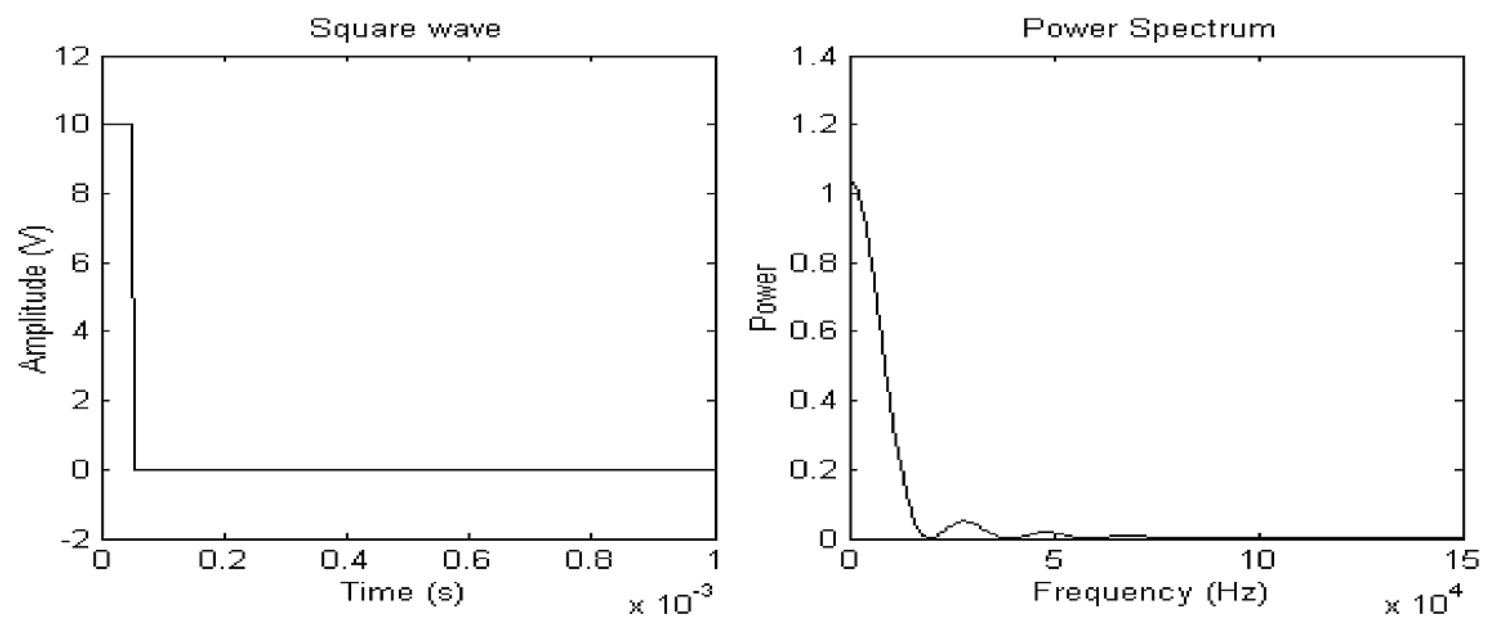

Fig. 6. Square actuation wave - time and frequency domain analysis.

or anti-symmetric waves can be controlled by bonding two PZTs in the same position in the plate, but in opposite surfaces. By actuating the PZTs in phase, symmetric waves generation is enhanced, while actuating the PZTs out of phase by $180^{\circ}$, anti-symmetric waves are more efficiently generated.

\section{Actuation wave}

As referred previously, in the computation of Lamb wave dispersion curves, one important conclusion derived from the dispersive behavior of Lamb waves is that, if multiple excitation frequencies are applied in the wave generation, packs of waves with different propagation velocities will be generated. It is then required a careful analysis of the actuation signal waveform to avoid the introduction of unnecessary excessive noise and added difficulty in the damage detection method. In the definition of the optimum waveform function, it is of the outmost importance to verify the frequency footprint of the actuation signal, by analyzing it in the frequency domain.

Several functions were investigated and the study of some of these waveforms will be presented in this section. When analyzing the functions in the frequency domain, the ideal one must present a well defined peak around the scanning frequency of interest. Such will provide the clear generation of a single pack of Lamb waves with a unique propagation velocity. Carefully selecting the scanning frequency, the clear separation between the different wave modes, in terms of their propagation velocity, can be achieved - so that clearly different wave mode arrival times to the sensors are verified, avoiding overlapping. Distinct times of flight (time between actuation and sensing, being related to the relative position and distance between actuator and sensors) present a reduced complexity in the sensor data, less noise, allowing for a clear separation and identification of the different modes. The accuracy and the definition of the relevant mode in the sensor signal are then increased.

At first it may have seemed that for a pulse echo technique it would be better to have an instantaneous or quasiinstantaneous actuation pulse, well defined in time, for example an impulse, ramp, or square wave. The wavelength would be small and the reflections would be easily identified. The problems generated by the application of those waveforms can be derived from the observation of their frequency domain spectrum. Due to very rapid changes in the waveform, a wide frequency band is excited, as presented in Figs 6 and 7. Different Lamb wave modes and multiple packs of waves with different propagation velocities would be emitted, resulting in a non clear, complex response, with multiple interferences, almost impossible to interpret.

Subsequently, once the importance in the definition of the waveform frequency was realized, a perfect sine function, finite in time, was analyzed. The frequency of the sine waveform was perfectly set and the analysis of the signal in the frequency domain revealed the predicted peak around the sine frequency, however it also revealed the existence of secondary peaks (lobes) around the known frequency harmonics - Fig. 8. 

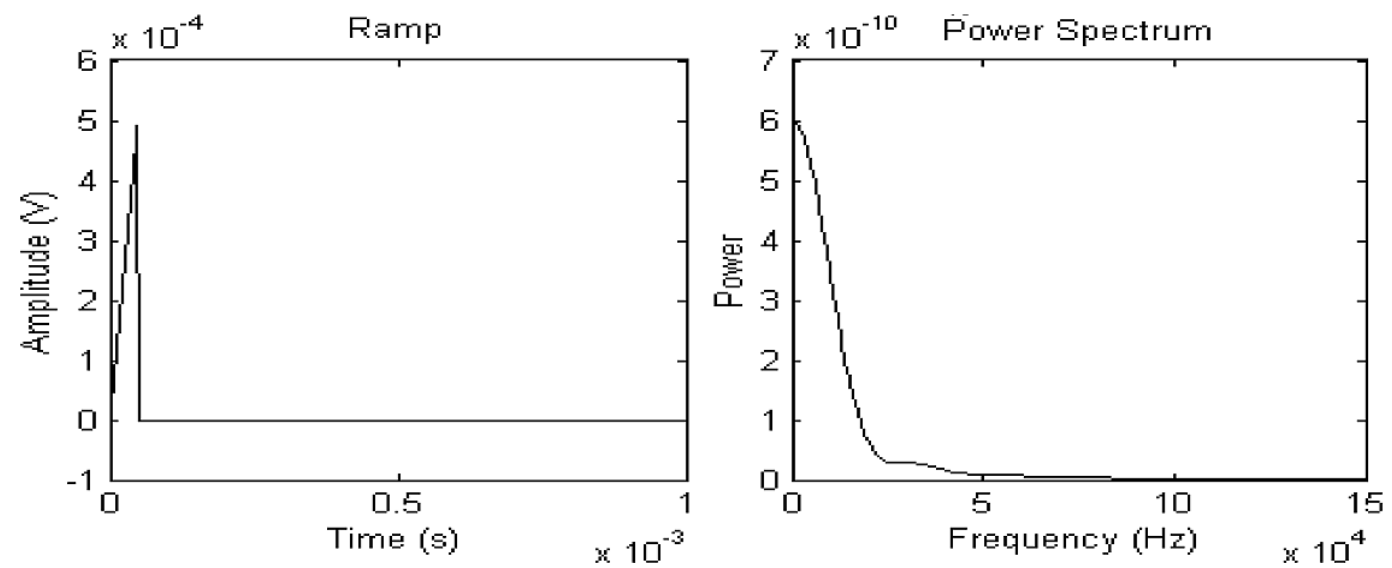

Fig. 7. Square actuation wave - time and frequency domain analysis.
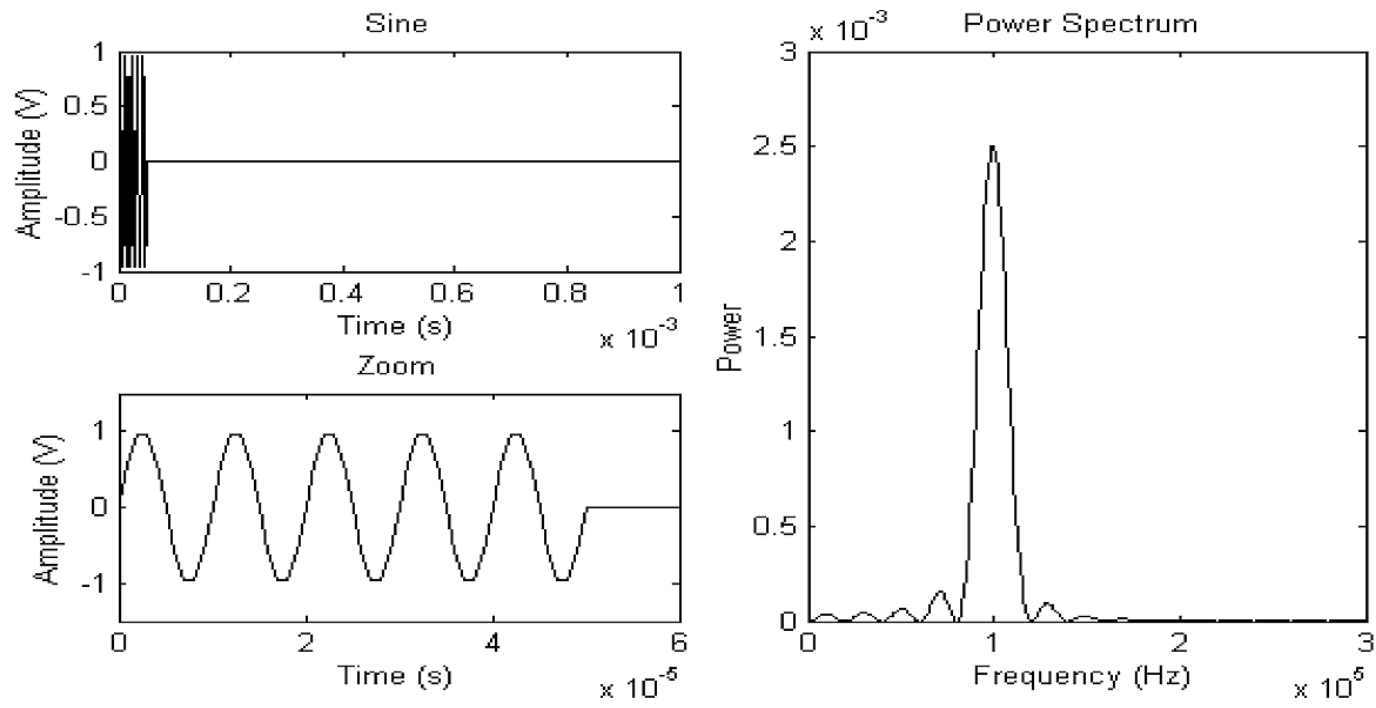

Fig. 8. Finite sine actuation wave - time and frequency domain analysis.

In order to disappear with the lobes, modulation was applied to the sine signal, in a similar fashion as a Hann window.

$$
f(t)=A \sin \left(2 \pi f_{t}\right) \sin \left(\frac{2 \pi f}{10} t\right)
$$

In Fig. 9 a distinct unique peak around the signal's selected frequency exists in the frequency domain analysis of such waveform - the sine frequency selected corresponds to the intended scanning frequency. Different ratios of sine to window frequencies were investigated. In terms of signal definition and frequency domain analysis results, a good compromise was attained for a selected sine frequency ten times the modulation window frequency, producing a signal with five peaks. The frequency domain signal was further optimized by smoothing the beginning and end of the actuation window.

\section{Experimental setup}

After performing several experiments using a preliminary experimental setup, just to assess the basics of Lamb wave emission, damage interaction and data acquisition, it was possible to determine the desired hardware and 

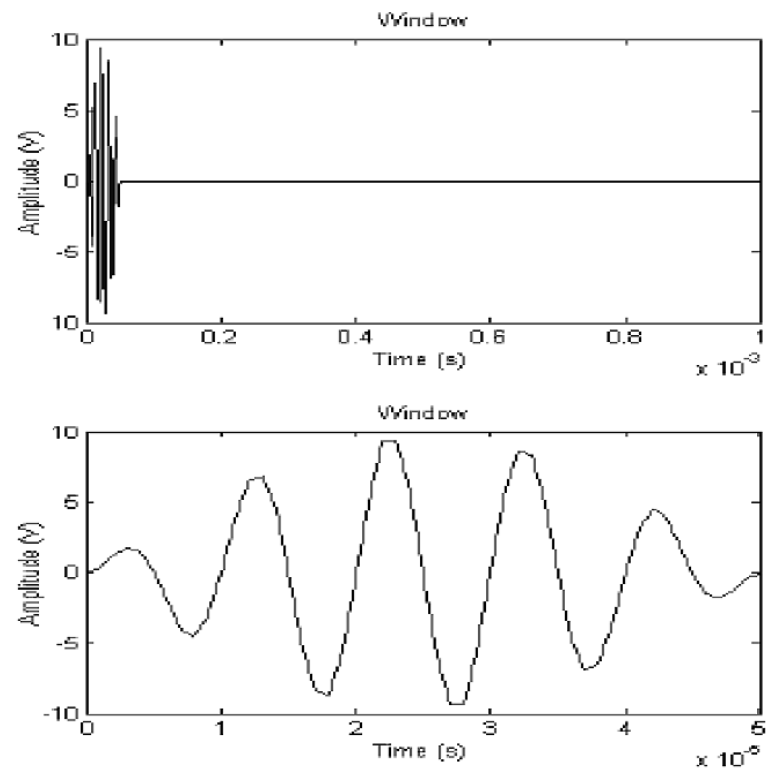

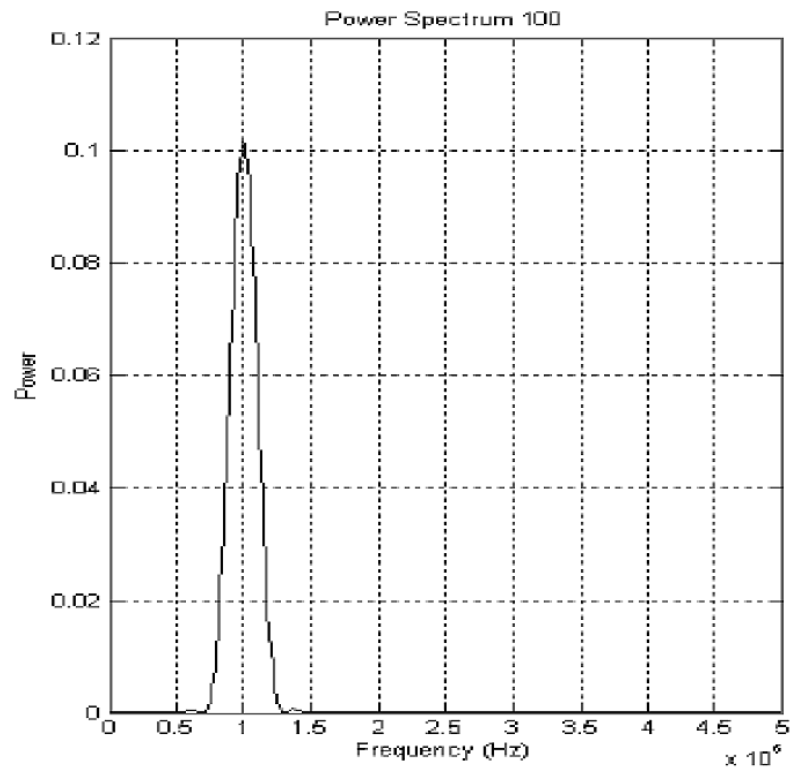

Fig. 9. Modulated sine actuation wave - time and frequency domain analysis.

software requirements to improve considerably the experimental setup. Essentially, the hardware should be able to handle high sampling frequencies while having enough memory to save all the necessary data acquired during each test, all with a suitable definition.

National Instruments provided boards complying with the requirements set. A NI PXI-5421 Arbitrary Waveform Generator, with $100 \mathrm{MS} / \mathrm{s}$ of sampling rate, 16bit of resolution and $8 \mathrm{MB}$ of memory was acquired. This board is particularly appropriate due to its high sampling rate and being an arbitrary waveform generator, allows the user to define completely the actuation signal - discrete, point by point, function definition, etc.

A NI PXI-5105, 8 channels Oscilloscope, with simultaneous sampling, 60MS/s of sampling rate and a 12Bit resolution, was also acquired. The two boards were mounted in a NI PXI-1033 chassis, connected to a Laptop using a PCI-Express card slot. The chassis and the connection to a computer were also carefully selected, not to limit the performance of the two boards.

As for the software, NI SIGNAL EXPRESS was initially used to assess the performance of the boards for application in a SHM system configuration, and simultaneously facilitate the implementation of such system. This software is extremely user friendly. It manages in a very simple way the actuation, the data acquisition and storage and finally performs some preliminary signal processing. At the same time, it supports a visual module with the capability of monitoring actuation and sensor signals in real time.

Since an in plane axisymmetric actuation is desired (with relation to the actuation source), the PZTs applied were of circular shape with through thickness d33. As experiments progressed, gradually the diameter of the transducers applied was reduced for the generation of higher frequency actuation waves (smaller wavelength), with increasing efficiency. As discussed, the amplitudes of the generated $\mathrm{S}_{0}$ and $\mathrm{A}_{0}$ waves were to be augmented if the PZT actuator diameter was equal to half or the total wavelength of the emitted wave, respectively. Also, the selected scanning frequency limited the choice of PZT transducers in terms of their natural frequencies' characteristics. The aluminum plate specimen used in the experiments had dimensions of $1 \mathrm{~m} \times 1 \mathrm{~m} \times 0.002 \mathrm{~m}$. A network of PZT transducers was bonded to the surface of one quarter of the plate.

The criteria for the selection of PZTs positions in the plate changed from the first to the second experimental setup. The main goal of the first setup was the generation of Lamb waves, confirmation of their propagation speed and to perform a crosscheck using the boundary expected reflections. Only two PZTs were necessary and they were placed as far apart and simultaneously as far from the plate boundaries as possible, in one quarter of the plate. The aluminum plate was laid on a foam layer to diminish possible wave reflections from the support contact points. 

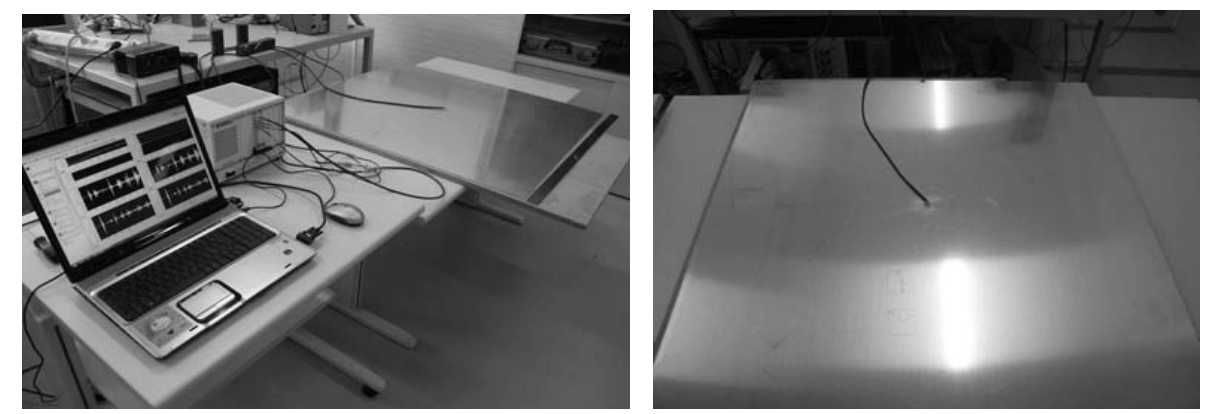

Fig. 10. Experimental setup.

The second setup was far more realistic. The plate was simply supported on all the boundaries, being the SHM system subjected to increasing external interference. In addition to the two PZTs used in the first setup, an additional transducer was bonded in the middle of the plate, since, for performing damage detection, a triangulation scheme had to be applied for the determination of damage position - three sensor signals are necessary.

\section{Frequency sweep}

To verify experimental setup limitations and reliability, several experiments were performed. Initially, a scanning frequency sweep, from a frequency of $20 \mathrm{kHz}$ to a frequency of $1 \mathrm{MHz}$, with a step of $20 \mathrm{kHz}$, was completed. Hardware limits were assessed and at first the computed dispersion curves were experimentally confirmed.

The previous conclusions were confirmed also through a detailed analysis of sensor signals resulting from the scanning frequency sweep - specifically considering the time separation between $S_{0}$ and $A_{0}$ and their amplitude variation with frequency. In Fig. 11 sensor signals are depicted for different scanning frequencies. For the sake of brevity only the more representative resultant sensor signals, corresponding to certain scanning frequencies, are presented.

As deduced from the dispersion curves, in the frequency range selected, up to 700kHZ, symmetric waves have a propagation velocity higher than anti-symmetric waves. In the sensor signals presented, the first wave (to the left, origin of times) is always the symmetric wave, while the second wave is the anti-symmetric mode. Concerning the frequencies at which $S_{0}$ and $A_{0}$ waves reached their highest amplitudes, the results attained confirm part of previously stated observations. The PZTs used in the scanning frequency sweep had a diameter of $8 \mathrm{~mm}$ and according to what was stated previously symmetric waves with a wavelength of $16 \mathrm{~mm}$ (double of PZTs diameter, i.e. being $8 \mathrm{~mm}$ half wavelength) would present higher amplitudes. Through the computed relation between wave frequency and wavelength presented in Fig. 5, a wavelength of $16 \mathrm{~mm}$ corresponded to a symmetric wave frequency of $340 \mathrm{kHz}$. As can be observed in Fig. 11, $\mathrm{S}_{0}$ presents the highest amplitude at that frequency. With $\mathrm{S}_{0}$ presenting higher amplitudes, its reflections are also expected to present higher amplitudes. As predicted, in the sensor signals acquired during the sweep, corresponding to frequencies around $340 \mathrm{kHz}$ - specifically between $260 \mathrm{kHz}$ and $420 \mathrm{kHz}$ -, the propagation of the boundaries' reflections of the symmetric wave mode can be clearly observed, just after the $\mathrm{A}_{0}$ wave (to the right, in the time scale).

With relation to anti-symmetric modes, it had to be considered also the fact that during these experiments, PZT transducers were bonded to only one of the sides of the specimen plate. Such had an effect in the wave mode generation efficiency along the frequency range elected. It was verified that for lower actuation frequencies, with the PZTs bonded to just one of the sides of the plate, there is a preferential generation of anti-symmetric waves presenting higher amplitudes. For higher generation frequencies the previously referred effect is not relevant and the symmetric wave mode becomes dominant. With this in mind, it was verified that $\mathrm{A}_{0}$ presented the highest amplitude for a scanning frequency of $140 \mathrm{KHz}$. As referred previously, by inspection of Fig. 2, the (generated and sensed) $\mathrm{A}_{0}$ wave should present its maximum amplitude when its wavelength equals the PZT diameter $(8 \mathrm{~mm})$. Examining Fig. 5, the $A_{0}$ highest amplitude (corresponding to a wavelength of $8 \mathrm{~mm}$ ) should have been obtained for a scanning frequency of around $200 \mathrm{kHz}$ (slightly higher than $140 \mathrm{kHz}$ ). 

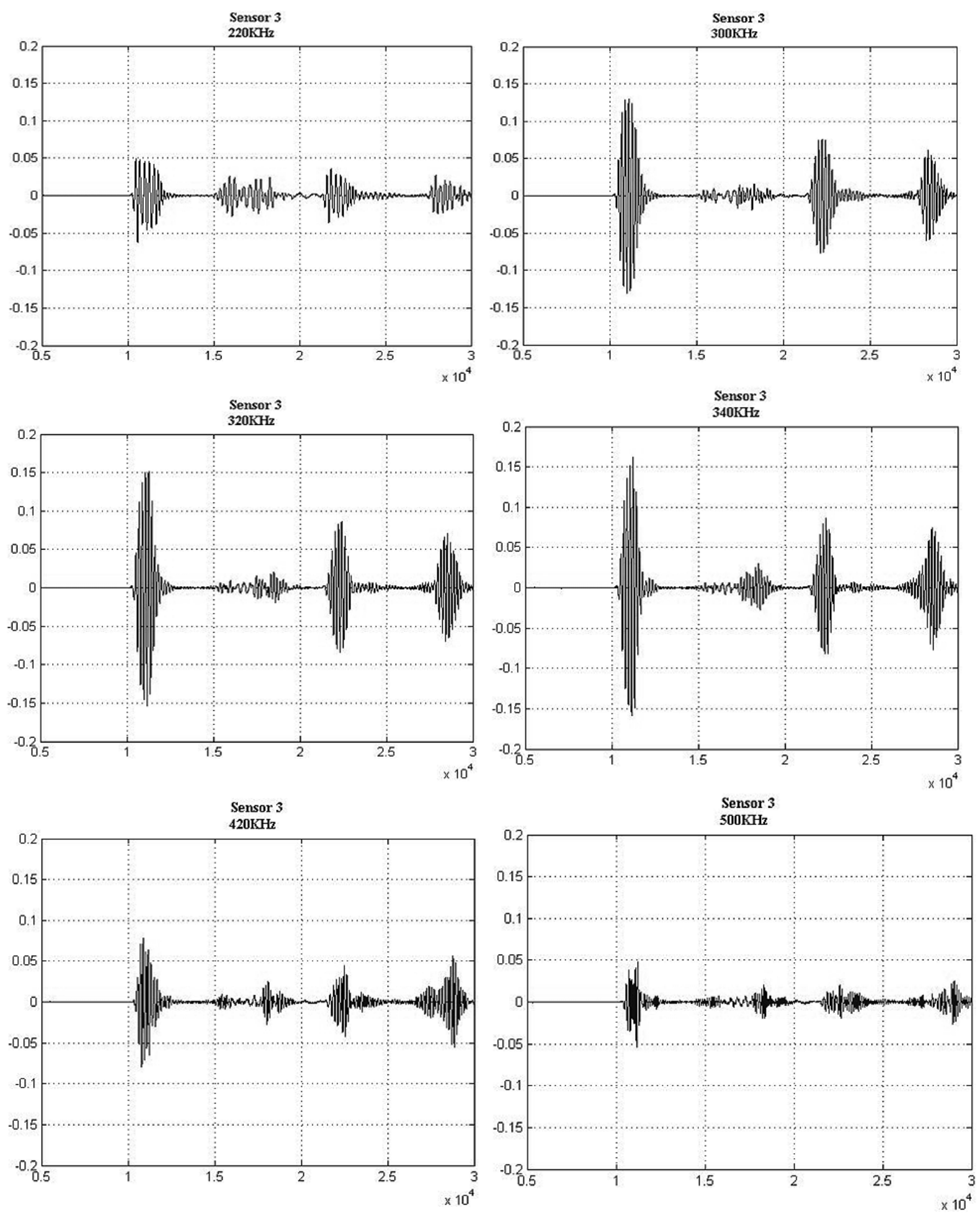

Fig. 11. Evolution of sensor signals for particular frequencies, during the initial frequency sweep, depicting propagating actuation waves $\mathrm{S}_{0}$, $\mathrm{A}_{0}$ and boundary reflections. 
Accordingly to the dispersion curves, in the sensor signals presented previously it is also observed that as the scanning frequency is increased during the sweep, the $S_{0}$ wave suffers a negligible shift to the right, meaning slightly increasing times of flight, or slightly decreasing propagation speeds (being almost constant for lower scanning frequencies). At the same time, the $\mathrm{A}_{0}$ wave is markedly shifted to the left during the sweep, with increasing scanning frequencies, i.e. lower times of flight or higher propagation speeds.

\section{Damage detection algorithm}

The applied algorithm for damage detection consists of the following steps:

- Select an actuation frequency and determine the wave's propagation velocity using the time of flight of the propagating actuation wave, between actuator and sensors, and confirmation of boundary reflections - dispersion curves.

- Execute one scan using a single PZT as actuator.

- Save the sensed response gathered by all PZTs.

- Repeat step 2 on the damaged structure.

- Repeat step 3. Now, for each PZT, both undamaged and damage profiles were obtained and saved.

- For each sensor, subtract the undamaged to the damaged data and determine the time at which differences are detected - the damage reflection occurred.

- Knowing the propagation velocity and the time between actuation and detected reflection, for each PZT, the distance traveled by the wave is determined. This distance is the sum of the distance between actuator and damage, and the distance between damage and sensor.

- For each PZT define an ellipse. The pair actuator PZT and sensor PZT positions are the two foci of the ellipse.

- For a selected coordinate system, solve the system of three equations (two ellipses and one circle) for the unknown damage position coordinates, i.e. determine the intersection of the ellipses.

The practical difficulties in the application of the method are related to the small amplitude of reflected waves - being difficult to detect - and the influence of noise in the sensor signal - creating false differences/reflections between damaged and undamaged signals.

The duration of a single scan (including a pausing time after its execution to allow for the damping of all reflections propagating in the plate) is less than $1 \mathrm{~ms}$. Also, the computation and storage capabilities required by the method are in the range to what is available presently in a common personal computer. The first obvious solution to decrease noise (random) influence and to obtain a better definition in the determination of the damage position, was to perform scans using all the different PZTs (in different positions) as actuators, one at a time for each scan, while the rest were used as sensors. Each scan was also repeated several times, both in undamaged and damaged states. To remove off tone noise, sensor signals were analyzed in the frequency domain and afterwards a second order band pass filter was applied, around the scanning frequency.

To process the considerable amounts of data gathered, fundamentally a noise threshold had to be set and consistent methods had to be developed. Besides analyzing directly the filtered signals, it was decided to apply concurrently several statistical methods including averaging of signals, determination of average highs and lows for all times and determination of signal bands. In this last case, no longer differences between damaged and undamaged correspondent signals were executed, but instead it was verified when the damaged corresponding signal band would exit the undamaged signal band.

Despite all these corrections, ghost damages, or better, ghost differences still existed. To avoid eliminating a true damage reflection, per each scan, the determination of multiple possible differences (potential damage reflections of different damages and positions) was allowed within a certain threshold, including the true damage and the false positives. All the combinations based on all the different potential differences were analyzed. Ellipses were formed and existence of ellipse intersections was verified. When intersections existed, their coordinates were saved, and the potential damage locations were plotted in the plate domain.

A code in LABVIEW, with embedded MATLAB codes, was developed to perform all the referred tasks (sequentially or not) in a fully automatic manner. In the determination of ellipse intersections an uncertainty area of 


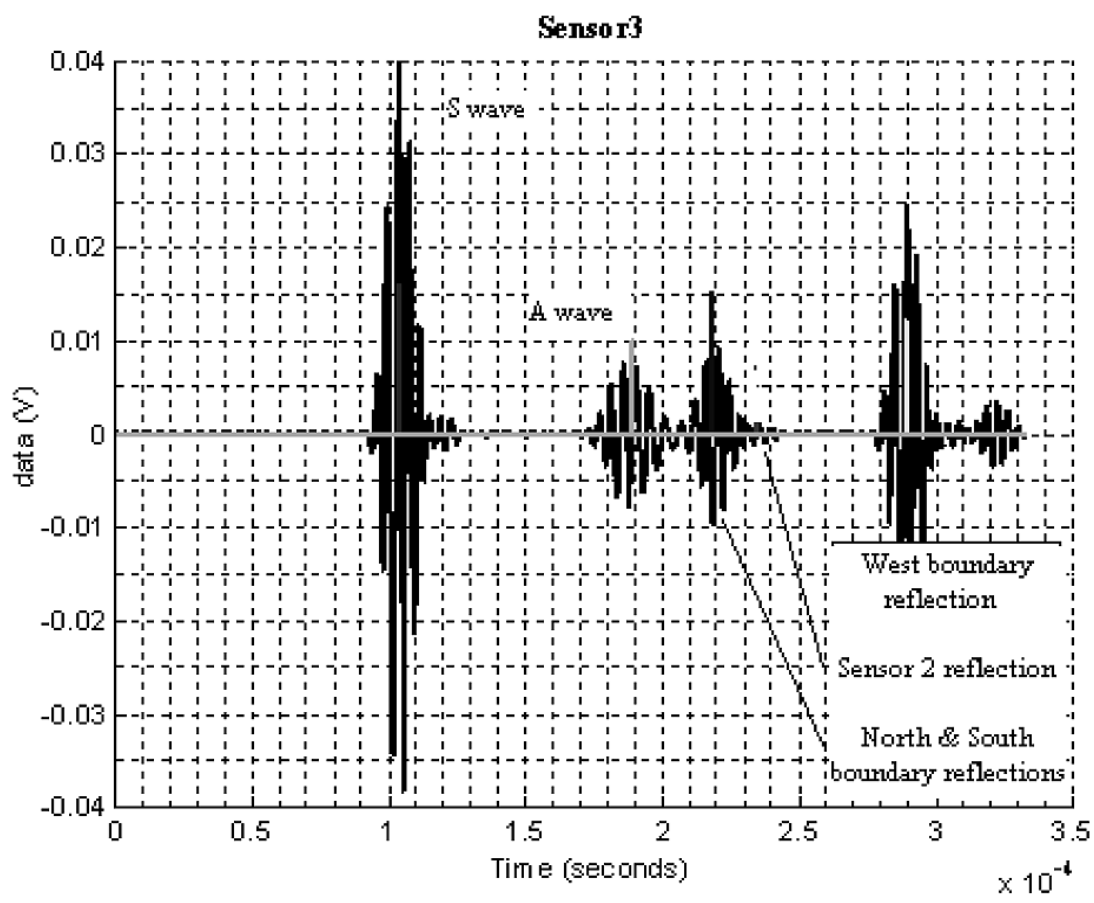

Fig. 12. Example of sensor signal for the undamaged plate showing boundary and other reflections.

$5 \mathrm{~mm}$ radius was considered around the intersection points. After plotting the possible intersection points the code allows for the user to select visually the damage location (the position with more intersections and particularly more intersections originating from different determined ellipses and different scans). Alternatively, if requested by the user, the program performs a probability analysis for all possible damages and respective determined locations. The final results are presented to the user, by plotting the $\mathrm{n}$ (defined by the user) most probable damage locations in the plate domain, with the elimination of the remaining false positives.

The LABVIEW program is also able to perform frequency scan sweeps to obtain dispersion curves.

\section{Results}

The inspection method was based in the $\mathrm{S}_{0}$ wave, since its propagation velocity is higher for the frequency range selected, it is easier to identify and less prone to noise (interference from $A_{0}$ wave, etc). As referred before $S_{0}$ wave and consequently its reflections reach their higher amplitudes for a frequency of around $340 \mathrm{kHz}$, while at that frequency the amplitude of the $\mathrm{A}_{0}$ wave is relatively small. A scanning frequency of $340 \mathrm{kHz}$ was then selected.

Experiments were performed with the setup presented and applying the developed software and damage detection scheme for the assessment of different types of damages. In the beginning, as referred before, sensor signal was analyzed to assess boundary and remaining transducers generated reflections. These are presented in Fig. 12.

All damages were successfully detected and their position was determined by the developed inspection system. The smallest cuts and holes had dimensions of $1 \mathrm{~mm}$. These were repeatedly introduced in different positions of the aluminum plate and with different orientations, in the case of the cuts, with relation to the PZT network. These damages were detected and their position was determined with a POD higher than $85 \%$, what is remarkable compared to the PODs of the current NDT\&E techniques, being used for practical inspection applications, for similar damage dimensions.

When cuts were oriented in a radial direction with relation to one of the PZTs, since their thickness was small (smaller than $1 \mathrm{~mm}$ ), the cut generated reflections detected by that particular PZT presented considerably small amplitudes. In a small number of the scans performed, these reflections fell within the noise threshold determined by 

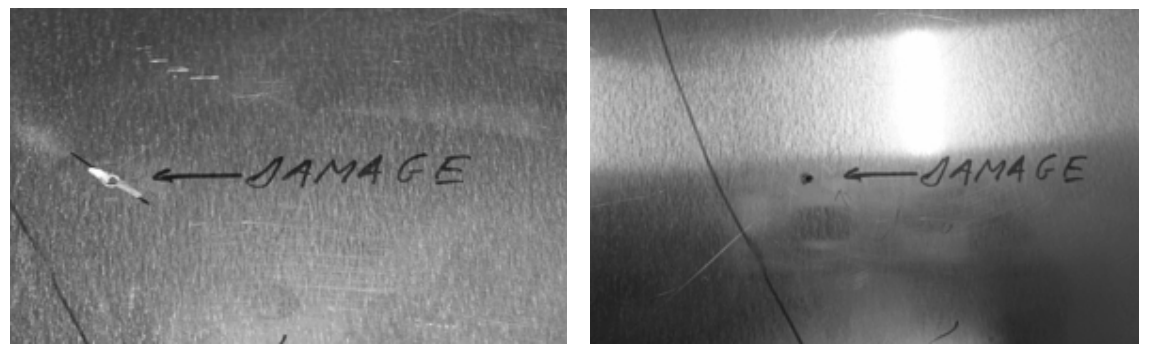

Fig. 13. Example of two types of damages created in the plate.

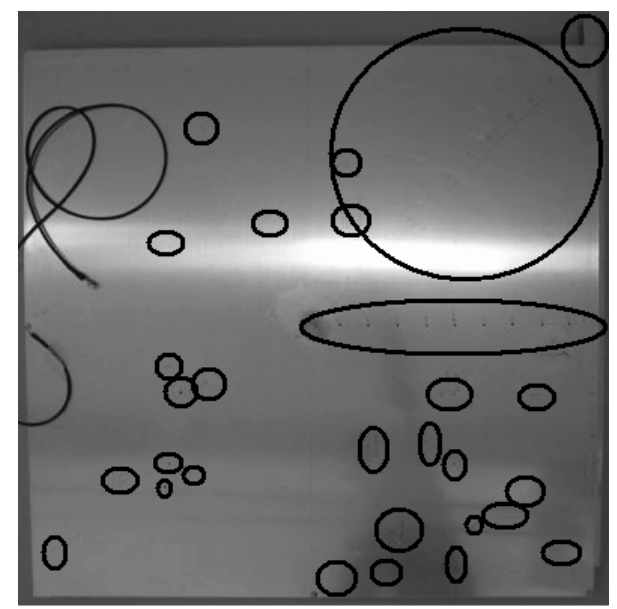

Fig. 14. Aluminum plate after all the executed experiments, with some of the damages marked (including a rivet hole line).

the software and were not easily detected by that particular transducer. However, since a network was implemented, it is impossible for the cut to be aligned with the radial directions of the three transducers of the network - being the worse case when it was aligned with two of the PZTs. Also, even if within the noise threshold determined by the software in particular scans, for one or two of the sensors signals, such reflections were usually detected, since the software allowed for the determination of multiple differences and possible reflections within each sensor signal. This problem was further minimized with the application of the filters and with the multiple repetitions of scans. Cuts farther from the network and oriented according to a radial direction centered in the center point of the network were also detected by the system. Due to the same reasons referred previously, after the execution of the final probability analysis, the system presented these damages as the most probable existing damages and respective locations, however with a smaller probability when compared to the other damages, locations and orientations experimented.

As referred, damages were created and detected in the plate sequentially and cumulatively. The software handled this difficulty since the sensors' data from different scans, executed at different times (in between the introduction of different damages), was recorded and could be used as the reference 'undamaged' state response signals, for comparison. Such allowed for damage growth monitoring and to detect new damages relatively to the ones existing at the time the reference scan was performed. Of course newer damages created behind existing damages with relation to the transducers network are considerably more difficult to detect.

At a later stage an $\mathrm{L}$ aluminum stringer $2 \mathrm{~mm}$ thick was also included (using the same aluminum plate specimen with all the damages created before). A single line of rivets was used to attach the stringer to the plate. Cuts with increasing dimension, initiating in a single rivet and also connecting adjacent rivets were introduced. Experiments to detect loosen rivets were also performed. In Fig. 14 the aluminum plate is presented after all the executed experiments. Some of the introduced damages are marked, as the rivet hole line, where the rivets attaching the stringer were placed. 

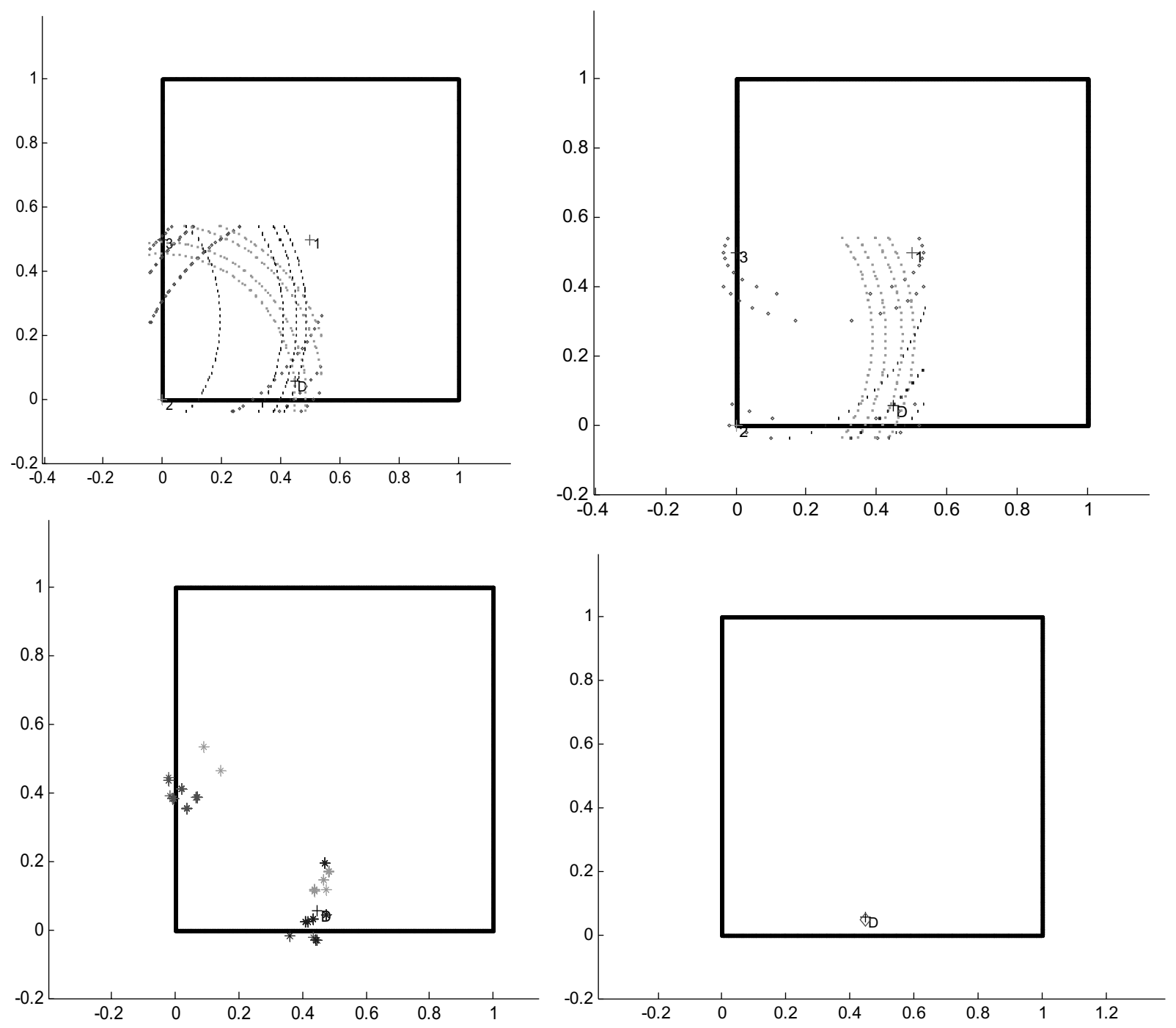

Fig. 15. Damage algorithm executed by the software - determination of most probable damage location (ellipses and intersections).

Cuts initiating in rivet holes and also the ones connecting adjacent rivets were successfully detected, as long as they are not behind the rivets with relation to the network. Specifically, cuts aligned with the network were only detected when their length was over $2 \mathrm{~mm}$, due to a minimum required time separation between their reflection and the contiguous rivet hole generated reflection. The minimum required separation time is dependent on the maximum sampling frequency of acquisition of the sensor signals by the oscilloscope (NI digitizer), in this case 60MS/s, and the wave propagation speed - and also on the minimum number of sampled points for the software reliably determine the existence of such reflection. Loosen rivets were also detect by this method. The positions of these types of damages (cuts originating in rivets, connecting adjacent rivets and loosen rivets) were determined with success, although it was verified a diminished precision in the determination of their radial position with relation to the network. Finally it was observed that, with the introduction of the stringer, damages created beyond it, with relation to the network, were not detected, since their reflections were destroyed (reflected) by the stringer and the rivets line.

For the smallest damage reliably detected, corresponding to a circular surface hole of $1 \mathrm{~mm}$ of diameter, the different steps in the process of determining the ellipses are presented in Fig. 15. Ellipses with foci in different pairs of PZT transducers are presented with different colors. These steps are executed after the detection of differences in the sensor signals between damaged and undamaged states. They are performed internally and automatically by 

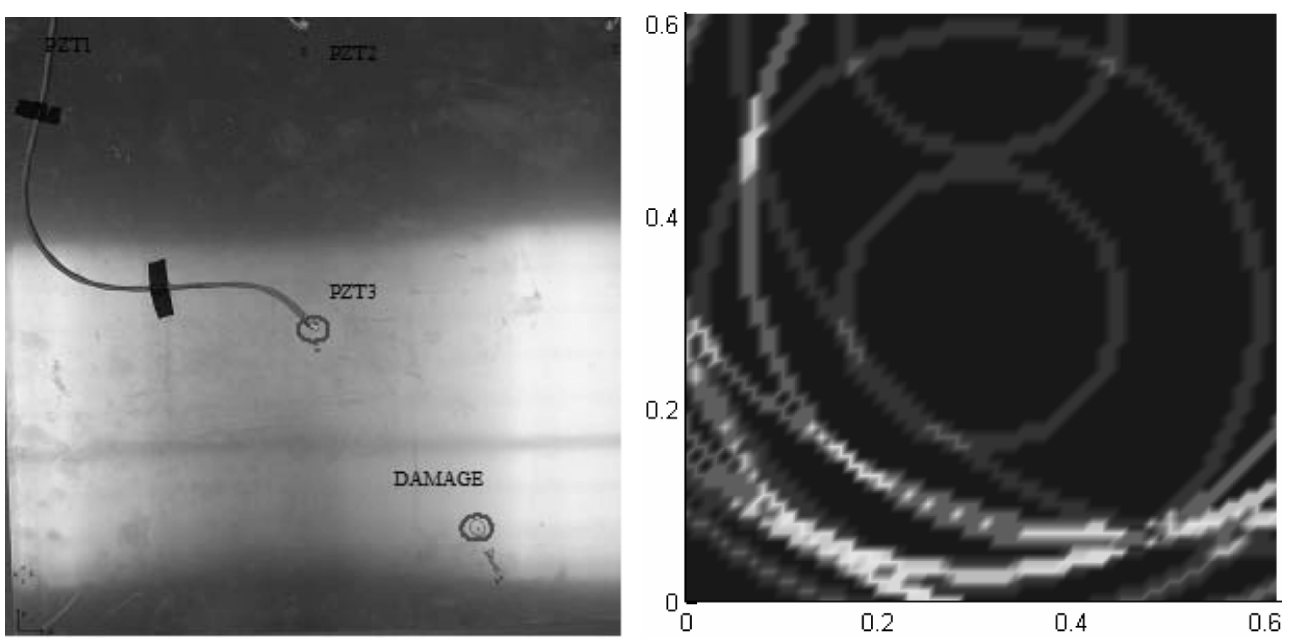

Fig. 16. Damage location.

the software and can be optionally presented to the user if that option is signaled in the beginning of scan execution. The different ellipses intersection points are plotted with different colors and finally, after the probability analysis executed by the software, the most probable existing damage position is indicated.

In Fig. 16, a zoom of the final screen presented by the software to the user is depicted, showing the most probable damage position. As for comparison it is presented a picture of the plate with the installed network and the indication of the location of the damage being detected and located.

The false positives were eliminated by the code, considering that the damage was located where the majority of potential damage positions fell. That is the location that presents a possible damage for all transducers' data and simultaneously the highest accumulation of different ellipse intersection points, presented with different colors.

In the determination of damage positions, a circumferential uncertainty area, centered in the location established by the system, with a $3 \mathrm{~mm}$ radius was verified for the presented inspection method, with the exception of damages related to rivets - uncertainty in the radial direction referred previously.

\section{Conclusions}

A Structural Health Monitoring methodology based on Lamb waves was developed. Based on an analysis of Lamb waves' dispersion curves, depicting the frequency dependence of the Lamb waves' propagation velocity and wavelength, an interesting range of scanning frequencies was determined. A discussion on dispersion curves was presented and methods to select the correct transducers and their dimensions were explained.

A detailed analysis of possible actuation signal waveforms to generate the actuation Lamb waves was also presented. By observing the frequency domain spectrum of the signals analyzed resulted in the selection of a modulated sine waveform. Due to the dispersive character of Lamb waves, an actuation signal exhibiting multiple frequencies is responsible for the generation of multiple wave packs with different propagation velocities. This in turn, results in a complex wave propagation pattern with the superposition of the different waves, and consequently in complex sensor signals, difficult to analyze, creating difficulties in the application of the inspection method. From the waveforms analyzed, the frequency domain spectrum of the latter was the only one that presented a clear single peak around the selected sine frequency - scanning frequency.

The requisites for the experimental equipment were determined and a PZT network was implemented in an aluminum plate. Preliminary experiments included a scanning frequency sweep to validate the computed dispersion curves. The frequency sweep permitted the determination of the tuned scanning frequency of $340 \mathrm{kHz}$.

A damage detection algorithm, based on the comparison of undamaged and damaged responses of the structure, was developed and implemented using MATLAB codes embedded in LABVIEW. Codes were developed for actuation generation and particularly for data post processing. A totally automated process was achieved. 
The simple algorithm implemented was successful in detecting and locating damage successfully in more than $85 \%$ of the damages created on the plate during the experiments. The accuracy and reliability of the method was dramatically increased by executing multiple consecutive tests since each scan is executed in less than $1 \mathrm{~ms}$ and then apply collaboratively different statistical methods for data post processing.

Surface and through the thickness cuts and circular and semi circular holes, as small as $1 \mathrm{~mm}$, were successfully and systematically detected. Damages were created sequentially and cumulatively in the whole aluminum plate specimen. The significance of such result is assessed when compared to the current NDT\&E results in terms of PoD and minimum detectable crack dimension.

Additionally, a stringer was attached to the plate by a single rivet line to simulate an aircraft skin structure. Cuts originating from rivet holes and connecting adjacent rivets, as well as loosened rivets were detected by the system. The introduction of the stringer resulted in a loss of precision in the determination of the radial position of the damages near it. Also, the network revealed significant difficulties in the detection of damages beyond the stringer.

\section{Acknowledgements}

The support given by the Portuguese Air Force, Instituto Superior T écnico, University of Victoria, Fundação para a Ciência e a Tecnologia, and the UVic Pacific Century Graduate Scholarship is acknowledged.

\section{References}

[1] D. Bical, NDT procedures qualification process, ATA NDT, Hartford, UK, 1995.

[2] H. Lamb, The Dynamical Theory of Sound, Edward Arnold, London, UK, 1910.

[3] J.W. Strutt, The Theory of Sound, Macmillan, London, UK, 1877.

[4] R. Mindlin, Higher frequency vibrations of crystal plates, Quarterly Applied Mathematics 19 (1961), 51-61.

[5] D.C. Worlton, Experimental confirmation of Lamb waves at megacycle frequencies, Journal of Applied Physics 32 (1961), $967-971$.

[6] J.L. Rose, A vision of ultrasonic guided wave inspection potential, Proceedings of the 7th ASME NDE Topical Conference, NDE 20 (2001), $1-5$.

[7] K. Worden, C.R. Farrar, G. Manson and G. Park, Fundamental Axioms of Structural Health Monitoring, 5th International Workshop in Structural Health Monitoring - IWSHM, Stanford-CA, USA, 2005.

[8] W. Hillger and U. Pfeiffer, Structural Health Monitoring Using Lamb Waves, 9th European Conference on Non-Destructive Testing ECNDT, Berlin, Germany, 2006.

[9] J.H. Nieuwenhuis, J.J. Neumann, D.W. Greve and I.J. Oppenheim, Generation and Detection of Guided Waves using PZT Wafer Transducers, IEEE Transactions on Ultrasonics, Ferroelectrics and Frequency Control 52(11) (2005), 2103-2111.

[10] K. Edalati, A. Kermani, M. Seiedi and M. Movafeghi, Defect Detection in Thin Plates by Ultrasonic LambWave Techniques, 8th International Conference of the Slovenian Society for Non-Destructive Testing Application of Contemporary Non-Destructive Testing in Engineering, Portoro, Slovenia, 2005.

[11] V. Giurgiutiu, Tuned Lamb Wave Excitation and Detection with Piezoelectric Wafer Active Sensors for Structural Health Monitoring, Journal of Intelligent Material Systems and Structures 16 (2005), 291.

[12] Y. Gomez-Ullate, F. Espinosa, P. Reynolds and J. Mould, Selective Excitation of Lamb Wave Modes in Thin Aluminium Plates using Bonded Piezoceramics: FEM Modeling and Measurement, Poster 205, European Conference on Non-Destructive Testing - ECNDT, 2006.

[13] N. Apetre, M. Ruzzene, S. Hanagud and S. Gopalakrishman, Nonlinear Spectral Methods for the Analysis of Wave Propagation, 49th AIAA/ASME/ASCE/AHS/ASC Structures, Structural Dynamics and Materials, Schaumburg-IL, USA, 2008.

[14] J.T. Chambers, B.L. Wardle and S.S. Kessler, Durability Assessment of Lamb Wave-Based Structural Health Monitoring Nodes, 47th AIAA/ASME/ASCE/AHS/ASC Structures, Structural Dynamics and Materials, Newport-RI, USA, 2006.

[15] G.S. Bottai, P.J. Pollock, T.A. Behling, V. Giurgiutiu, S.M. Bland and S.P. Joshi, Damage Detection in Cryogenic Composites for Space Applications Using Piezoelectric Wafer Active Sensors, 49th AIAA/ASME/ASCE/AHS/ASC Structures, Structural Dynamics and Materials, Schaumburg-IL, USA, 2008.

[16] G. Park, H. Sohn, C.R. Farrar and D.J. Inman, Overview of Piezoelectric Impedance-Based Health Monitoring and Path Forward, The Shock and Vibration Digest 35(6) (2003), 451-463.

[17] V. Giurgiutiu and J. Bao, Embedded Ultrasonic Structural Radar With Piezoelectric Wafer Active Sensors for the NDE of Thin-Wall Structures, ASME International Mechanical Engineering Congress, New Orleans-LA, USA, 2002.

[18] R.P. Dalton, P. Cawley and M.J. Lowe, The Potential of Guided Waves for Monitoring Large Areas of Metallic Aircraft Fuselage Structure, Journal of Nondestructive Evaluation 20(1) (2001), 29-46.

[19] J.E. Michaels and T.E. Michaels, Guided wave signal processing and image fusion for in situ damage localization in plates, Wave Motion 44 (2007), 482-492.

[20] J. Ihn and F. Chang, Pitch-catch Active Sensing Methods in Structural Health Monitoring for Aircraft Structures, Structural Health Monitoring 7(1) (2008), 5-15. 
[21] X. Zhao, H. Gao, G. Zhang, B. Ayhan, F. Yan, C. Kwan and J.L. Rose, Active health monitoring of an aircraft wing with embedded piezoelectric sensor/actuator network: I. Defect detection, localization and growth monitoring, Smart Materials and Structures 16 (2007), $1208-1217$.

[22] I. Read, P. Foote, J. McFeat and Y. MacPheat, Flight Trials of Structural Damage Detection Systems on a Fast Military Jet, 4th European Workshop in Structural Health Monitoring - EWSHM, Krakow, Poland, 2008. 

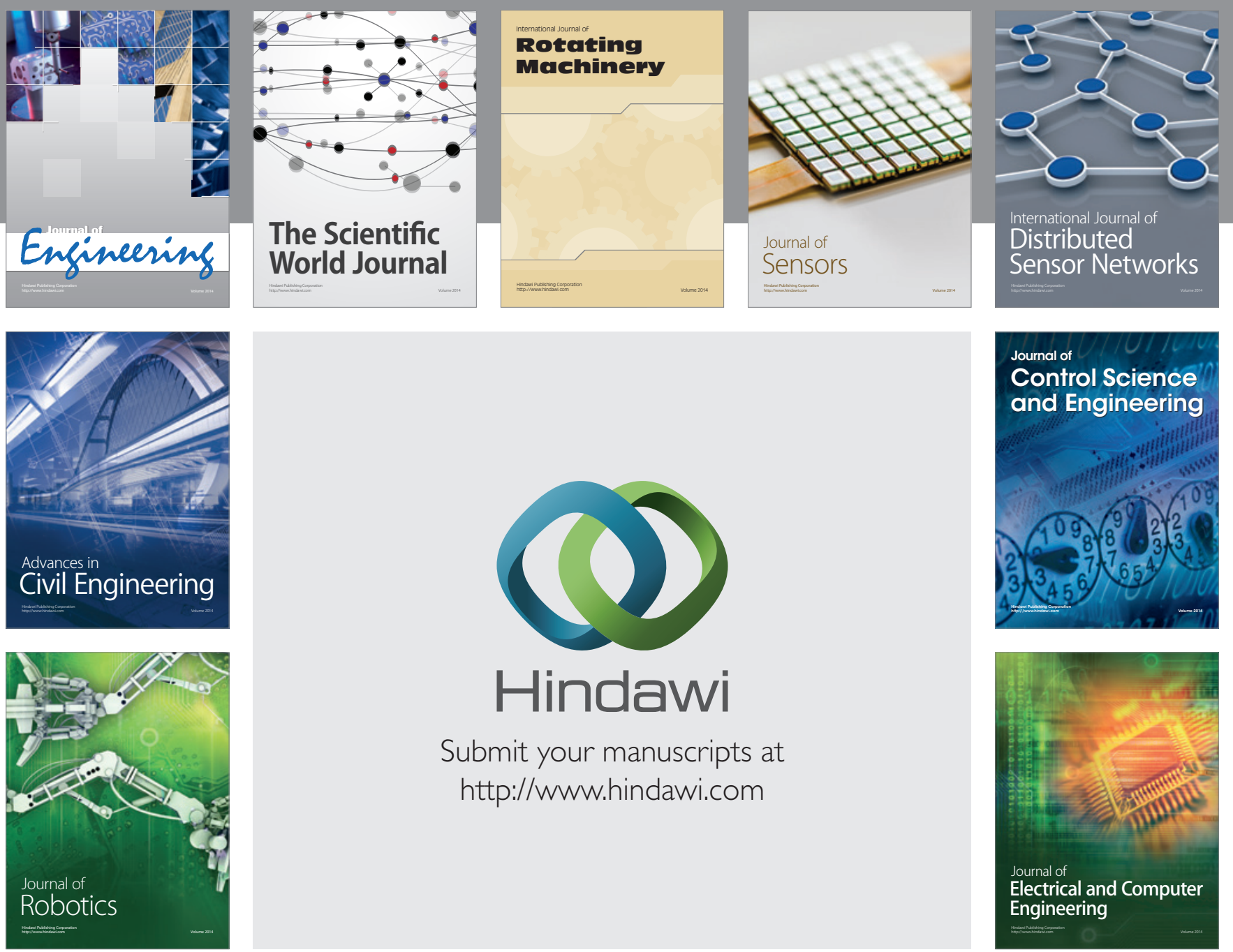

Submit your manuscripts at

http://www.hindawi.com
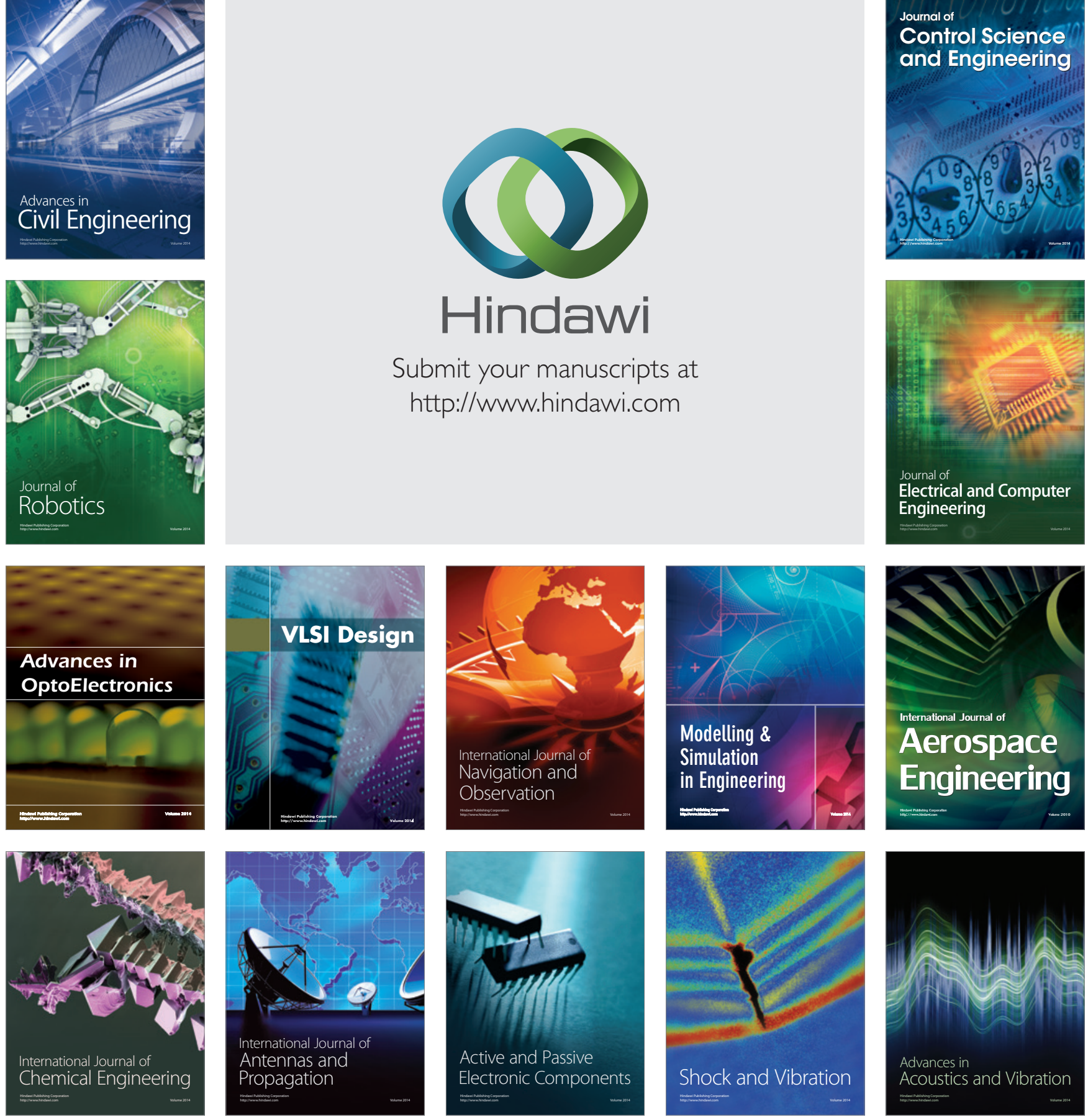